\title{
BMJ Open Investigating the efficacy and safety of elobixibat, an ileal bile acid transporter inhibitor, in patients with Parkinson's disease with chronic constipation: a multicentre, placebo-controlled, randomised, double-blind, parallel- group study (CONST-PD)
}

\author{
Taku Hatano (iD , , Genko Oyama (1) , ${ }^{1}$ Yasushi Shimo (1) , ${ }^{2}$ Kotaro Ogaki, ${ }^{3}$ \\ Noriko Nishikawa, ${ }^{1}$ Jiro Fukae, ${ }^{2}$ Ryota Nakamura, ${ }^{3}$ Naohide Kurita, ${ }^{3}$ Taiji Tsunemi, \\ Yutaka Oji, ${ }^{1}$ Shinji Saiki, ${ }^{1}$ Kenya Nishioka, ${ }^{1}$ Haruka Takeshige-Amano, ${ }^{1}$ \\ Daisuke Taniguchi, ${ }^{1}$ Takashi Ogawa (D) , ${ }^{1}$ Hikaru Kamo, ${ }^{1}$ Hiroto Eguchi, ${ }^{2}$ \\ Atsuhito Fuse, ${ }^{2}$ Asuka Nakajima, ${ }^{2}$ Masayoshi Kano, ${ }^{2}$ Sho Nakajima, ${ }^{3}$ \\ Naotake Yanagisawa, ${ }^{4}$ Nobutaka Hattori ${ }^{1}$
}

To cite: Hatano T, Oyama G, Shimo $\mathrm{Y}$, et al. Investigating the efficacy and safety of elobixibat, an ileal bile acid transporter inhibitor, in patients with Parkinson's disease with chronic constipation: a multicentre, placebo-controlled, randomised, double-blind, parallel-group study (CONST-PD). BMJ Open 2022;12:e054129. doi:10.1136/ bmjopen-2021-054129

- Prepublication history for this paper is available online. To view these files, please visit the journal online (http://dx.doi. org/10.1136/bmjopen-2021054129).

Received 04 June 2021

Accepted 26 November 2021

Check for updates

(C) Author(s) (or their employer(s)) 2022. Re-use permitted under CC BY-NC. No commercial re-use. See rights and permissions. Published by BMJ.

For numbered affiliations see end of article.

Correspondence to Dr Nobutaka Hattori; nhattori@juntendo.ac.jp and Dr Taku Hatano;

thatano@juntendo.ac.jp

\section{ABSTRACT}

Introduction Chronic constipation worsens the quality of life (QOL) of patients with Parkinson's disease (PD). Elobixibat, an ileal bile acid transporter inhibitor, is a useful laxative, but its effect on chronic constipation in patients with PD remains unclear. Therefore, we designed a placebo-controlled, randomised, double-blind study to investigate the efficacy and safety of elobixibat in patients with PD with chronic constipation.

Methods and analysis The study will consist of 2-week observation and 4-week treatment periods. Patients with clinically established PD will record the status of spontaneous bowel movements and use of rescue medications/concomitant medications in a Bowel Movement Diary from the start of the observation period at visit 1 (week -2). At visit 2 (week 0), patients will be assessed for final registration based on the diary records and physical examinations, and allocated to either the elobixibat or placebo group. Daily intake of the investigational drug will be recorded in the diary. Patients will undergo laboratory tests and answer constipationrelated, PD-related and QOL-related questionnaires at visits 2 and 4 (week 4). Subjective symptoms and objective findings will be collected at visits 2, 3 (week 2) and 4 . Since patients' motor function might be improved by treatment of constipation, the use of dopamine preparations will also be monitored. Bowel movement data and other parameters will be compared between groups. Safety information will be collected as adverse events, specifically focusing on those occurring in association with study conduct.

Ethics and dissemination This study will be conducted in accordance with the Helsinki Declaration, the Clinical Trials Act of the Japan Ministry of Health, Labour and
Strengths and limitations of this study

- The key strength of this study is its design as a randomised, double-blind, placebo-controlled trial to determine the efficacy and safety of elobixibat for Parkinson's disease patients with chronic constipation.

- A further strength is that it will examine not only the efficacy of elobixibat for Parkinson's disease (PD)related constipation, but also its effects on quality of life and movement in patients with PD.

- Because of the short study period (4-week administration), the long-term efficacy and safety of elobixibat may not be clarified.

- Bowel movement diary data might have limited credibility because they are based on the patients' subjective responses.

Welfare, and related laws and regulations. The study was approved by the Juntendo University Certified Review Board. The results will be disseminated through an online study registry (Japan Registry of Clinical Trials), presented at scientific conferences, and published in medical journals.

Trial registration number JPRN-jRCTs031200172; Preresults.

\section{INTRODUCTION}

Parkinson's disease (PD) is one of the most common neurodegenerative disorders. Whereas PD is commonly considered a movement disorder, patients with PD have many 
difficulties with non-motor symptoms, such as dementia, anxiety, sleep disturbance, and autonomic dysfunction. ${ }^{1}$ Chronic constipation is one of the common digestive complications experienced by patients with PD even before or at an early stage of disease progression, with its frequency varying from $20 \%$ to $70 \%$ depending on the definition. ${ }^{2-6}$ Since long-lasting constipation may decrease quality of life (QOL) and be occasionally accompanied by life-threatening intestinal perforation and/or megacolon syndrome, it should be appropriately controlled. ${ }^{78}$

Multiple types of laxatives are conventionally available for the treatment of chronic constipation, including bulkforming agents such as carmellose sodium and dioctyl sodium sulfosuccinate, stimulants such as sennoside and sodium picosulphate hydrate, and osmotic agents such as cathartic salts (magnesium oxide and magnesium sulphate). While stimulants and cathartic salts have been widely used, stimulants may cause intractable constipation via drug resistance if used consecutively for a long duration, and magnesium oxide may cause hypermagnesaemia in elderly patients and patients with kidney malfunction. ${ }^{9}$

Recently, in addition to existing osmotic agents (macrogol 4000 often in combination with electrolytes, movicol, lactulose), new classes of laxatives have been developed for chronic constipation, including epithelial function transformation drugs (lubiprostone, linaclotide) and an ileal bile acid transporter (IBAT) inhibitor (elobixibat).

However, the evidence for the effectiveness of laxatives either conventional or recently developed for the treatment of chronic constipation in patients with PD is not extensive, except for macrogol and lubiprostone, a chloride channel-2 activator, which have been shown to be significantly efficacious over placebo in improving bowel movement frequency. ${ }^{10}{ }^{11}$ Although probiotics and prebiotic fibre were also proposed to be useful for the treatment of PD-related constipation, ${ }^{12}{ }^{13}$ more evidence for different classes of laxatives is needed to expand therapeutic options for chronic constipation in patients with PD.

Elobixibat is an inhibitor of IBAT, which is expressed in the distal ileum. Bile acids escape the reabsorption process via the action of this drug and then enter the large intestinal lumen. ${ }^{14}$ The increased levels of bile acids may interact with transmembrane G-protein-coupled receptor (TGR5) molecules, leading to an influx of water and electrolytes into the lumen. The bile acid-TGR5 interaction also triggers serotonin release into the intestinal wall, which activates intrinsic primary afferent neurons. This leads to an interneuron-mediated activation of motor neurons that finally activates large-intestinal motility, ${ }^{15}$ inducing colonic high-amplitude propagated contractions. ${ }^{16}$ Recently, a large-scale, multicentre, randomised, double-blind phase 3 study was conducted, revealing that elobixibat resolved idiopathic chronic constipation with no serious safety concerns. ${ }^{17}$ Elobixibat is thus expected to be a novel option for the treatment of chronic constipation on the basis of its mode of action being totally different from those of the other existing laxatives. ${ }^{18} 19$

We are planning to conduct a study to examine the efficacy and safety of elobixibat in patients with PD with chronic constipation. The superiority of elobixibat over placebo will be explored in a randomised, double-blind, comparative study during 4-week daily administration of the drug to eligible patients. The drug will also be evaluated for its safety and potential impact on the underlying PD condition. The study methodology is detailed in this article.

\section{METHODS AND ANALYSIS}

The objective of this study is to explore a novel therapeutic option for chronic constipation in patients with PD. The conduct of this study was approved by the Juntendo University Certified Review Board.

\section{Primary endpoints}

The weekly frequency of spontaneous bowel movements with no assistance from rescue therapies such as bisacodyl suppositories, enema, disimpaction, etc (see below) will be recorded during each 7-day segment of the treatment period (week 0-1 up to week 3-4; see figure 1). Bowel movements observed within 24 hours of suppository use will not be counted. Changes in the frequency at visit 4 (week 4) from baseline (visit 2 /week 0; see figure 1) will be compared between the elobixibat group and the placebo group.

\section{Secondary endpoints}

Weekly changes from baseline in the frequency of spontaneous bowel movements and complete spontaneous bowel movements (ie, no sensation of incomplete evacuation) will be assessed throughout the treatment period (visit 4/week 4; figure 1). Changes from baseline in stool form will also be assessed up to week 4 using the Bristol Scale. ${ }^{20}$

Use of rescue medication and questionnaire surveys using the Japanese version of Patient Assessment of Constipation Quality of Life (JPAC-QOL) ${ }^{21}$ Movement Disorder Society-Unified Parkinson's Disease Rating Scale (MDSUPDRS), ${ }^{22}$ Parkinson's Disease Questionnaire-39 (PDQ$39),{ }^{23}$ and Euro-Qol 5 dimension-5 level (EQ-5D-5L) ${ }^{24}{ }^{25}$ will be assessed. The use of dopamine preparations will also be monitored, since the effectiveness of elobixibat in improving constipation via increased levels of intestinal bile acids may also improve small intestinal absorption of the anti-Parkinsonian medications.

Subgroup analyses will be further performed for these endpoints by the presence or absence of complications, age ( $\geq$ or $<65$ years), Hoehn and Yahr scale (1-4), duration of the underlying disease (PD; $\geq$ or $<$ median), dose equivalence of L-Dopa prior to elobixibat initiation, and duration of chronic constipation ( $\geq$ or $<20$ years). 


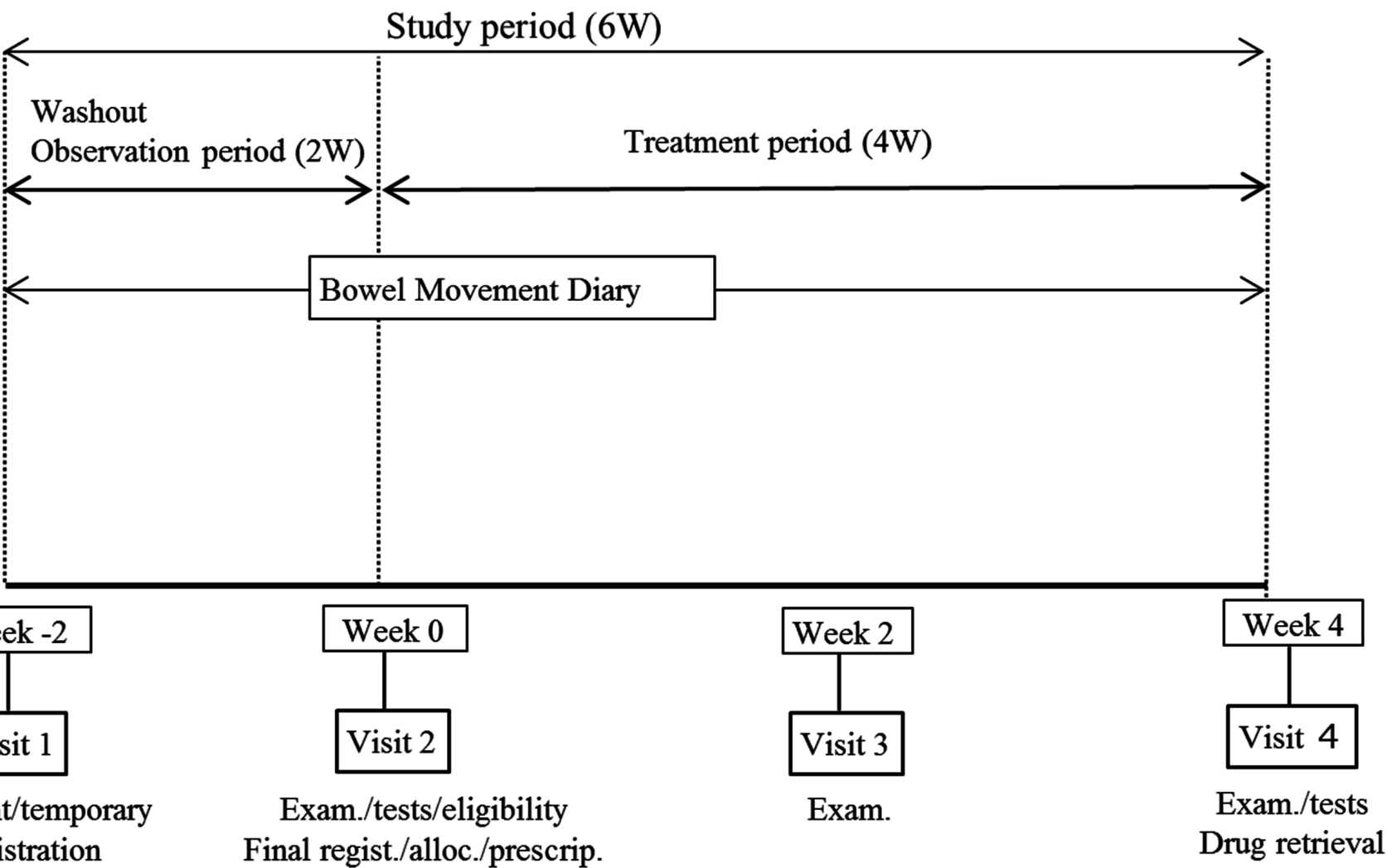

\section{Consent/temporary} registration Final regist./alloc./prescrip.

Figure 1 Study outline. Wash-out: previous medications except for anti-parkinsonian medications will be washed out during the observation period. Consent: written, informed consent exam. Physical examinations test: laboratory tests eligibility: eligibility confirmed for study participation Alloc. Patient allocation to either treatment with elobixibat or with placebo Prescrip. Prescription of the investigational medication according to patient allocation drug retrieval: unused/remaining investigational medications will be retrieved.

\section{Overall study design}

This study is designed as a multicentre, randomised, double-blind, placebo-controlled, parallel-group study at three academic centres (Juntendo University Hospital, Juntendo University Urayasu Hospital and Juntendo University Nerima Hospital), in which patients with PD with chronic constipation will preprandially receive elobixibat (Goofice; EA Pharma, Tokyo, Japan) or its indistinguishable matched placebo once daily for 4 weeks, and the frequency of spontaneous bowel movements will be compared between the patients receiving either elobixibat or placebo. Safety information, including adverse events (AEs) and discontinuation and interruption of the investigational drugs, will also be collected.

The study will consist of two periods, the observation period and the treatment period. It will be conducted from 26 October 2020 (date of the first announcement in the Japan Registry of Clinical Trials; jRCT) through 30 September 2022. As seen in figure 1, we plan two steps for patient registration, temporary and final. At week -2 (visit 1) of the observation period, patients with PD will be provided with detailed information about the study. After providing written, informed consent for study participation, they will be temporarily registered based on the patient definitions (see below). Following the observation period, the patients will be further assessed for study participation at week 0 (visit 2). Once none of the exclusion criteria are confirmed to be applicable, the patients will be finally registered and assigned to either the elobixibat group or the placebo group in a doubleblind manner (figure 1). The eligible patients will visit their clinical sites twice more (visit 3 at week 2 and visit 4 at week 4) during the 4-week treatment period.

A bowel movement diary will be provided at week -2 (visit 1, see below) to each patient, once temporarily registered. The patient will record drug use, bowel movements, etc in the diary daily throughout the study period.

\section{Patient definitions}

Patients will be diagnosed as having PD in reference to the diagnostic criteria defined by the International Parkinson and Movement Disorder Society ${ }^{26}$ and must be in stages $1-4$ on the Hoehn and Yahr scale. ${ }^{27}$ The diagnosis of chronic constipation will be made referring to the Rome IV-defined criteria. ${ }^{28}{ }^{29}$ Outpatients aged $\geq 20$ years at the time of informed consent must have two or more of the following symptoms related to spontaneous bowel movements from at least 6 months before consent: $(1)<3$ spontaneous bowel movements per week; (2) straining frequency $>25 \%$ of defecations; (3) frequency of lumpy or hard stools $>25 \%$ of defecations and (4) sensation of incomplete evacuation $>25 \%$ of defecations. Patients who meet these criteria will be temporarily registered, once written, informed consent is provided (figure 1). 
A patient with PD will be excluded at temporary registration if he/she: has (or is suspected to have) organic constipation or dyschezia; is unable to use a rescue medication (bisacodyl $10 \mathrm{mg}$ suppositories; Teleminsoft Suppositories $10 \mathrm{mg}$, Cox Japan, Tokyo, Japan); currently has serious kidney dysfunction (creatinine $\geq 2.00 \mathrm{mg} / \mathrm{dL}$ ), serious hepatic dysfunction (total bilirubin $\geq 3.0 \mathrm{mg} / \mathrm{dL}$, or AST or ALT $\geq 100 \mathrm{U} / \mathrm{L}$ ), or serious cardiac dysfunction; has malignant tumour(s); has a history of hypersensitivity to elobixibat; is taking bile acid preparations (ursodeoxycholic acid, chenodeoxycholic acid), aluminiumcontaining preparations (sucralfate hydrate, aldioxa, etc), cholestyramine, colestimide, digoxin, dabigatran etexilate mesylate or midazolam; has a Mini-Mental State Examination score ${ }^{30} \leq 26$; or is considered ineligible by the investigator or subinvestigator, hereinafter termed (sub) investigator, for study participation because of safety concerns, poor protocol compliance, etc. Female patients will also be excluded if they are pregnant, breast feeding or expecting pregnancy during the study period.

Patient exclusion is further scheduled at the final registration if one of the following cases applies to a patient during the 2-week observation period: use of the rescue medication (bisacodyl $10 \mathrm{mg}$ suppositories) $\geq 5$ times; use of the rescue medication within 72 hours postbowel movement; experience of spontaneous bowel movement with mushy or watery stool according to Bristol Stool Form Scale type 6 or $7 ;^{20}$ or use of prohibited medications/therapies (see below).

\section{Study procedures and schedule}

Registration of study patients and their allocation to the investigational drug will be performed via an internetmediated Interactive Web Response System (IWRS). The (sub)investigator (or study-assigned coordinator) will access the specific website for patient registration and allocation using a specific ID and password, and complete the necessary information about each anonymised patient. Investigational drug allocation will be instantly presented for each patient following eligibility judgement (see below).

The schedule of this study is shown in figure 2.

The (sub)investigator will give detailed explanations of this study to each patient with PD who meets the inclusion criteria but not the exclusion criteria at visit 1 and then obtain the patient's written, informed consent on a free will basis. The patient will be informed that he/ she may withdraw his/her consent for any reason and at

\begin{tabular}{|c|c|c|c|c|c|}
\hline \multirow[b]{2}{*}{ Visit } & & \multirow{2}{*}{$\frac{\text { Observation period }}{\text { Visit } 1}$} & \multirow{2}{*}{$\frac{\text { Administration start }}{\text { Visit } 2}$} & \multicolumn{2}{|c|}{ Treatment period } \\
\hline & & & & Visit 3 & Visit 4 \\
\hline $\begin{array}{l}\text { Date } \\
\text { (Allowance*) }\end{array}$ & & 14 days prior to Visit 2 & $\begin{array}{c}\text { Day } 1 \\
(\leq 5 \text { days post-Visit } 2)\end{array}$ & $\begin{array}{l}\text { Day } 15 \text { post Visit } 2 \\
(\leq \pm 7 \text { days }))\end{array}$ & $\begin{array}{c}\text { Day } 29 \text { post Visit } 2 \\
\text { ( } \leq 7 \text { days) or discontinuation }\end{array}$ \\
\hline Informed consent & & $\bigcirc$ & & & \\
\hline Eligibility confirmation & & $\begin{array}{c}\bigcirc \\
\text { (Temp. regist, } \dagger)\end{array}$ & $\stackrel{\bigcirc}{\circ}$ & & \\
\hline \multirow{5}{*}{ Physical examinations } & Patient background & O & & & \\
\hline & Subjective symptoms/objective findings & & O & O & 0 \\
\hline & History/complications & O & O & & \\
\hline & Physical examinations & $\bigcirc$ & 0 & & 0 \\
\hline & Vital signs (blood pressure/pulse rate) & & 0 & & 0 \\
\hline \multirow{3}{*}{ Bowel Movement Diary } & Use of investigational medication & & 4 & & $\rightarrow$ \\
\hline & Use of rescue/concomitant medication & & & & $\rightarrow$ \\
\hline & Other therapy for constipation & & & & \\
\hline Adverse events & & $\longleftarrow$ & & & $\rightarrow$ \\
\hline JPAC-QOL & & & O & & $\mathrm{O}$ \\
\hline MDS-UPDRS & & & O & & O \\
\hline PDQ-39 & & & O & & O \\
\hline EQ-5D & & & 0 & & 0 \\
\hline \multirow{2}{*}{ Laboratory tests } & Haematology & & O & & O \\
\hline & Blood chemistry & & 0 & & 0 \\
\hline
\end{tabular}

Figure 2 Study schedule* *Allowance denotes the time window allowed relative to the date of visit 1 for visit 2 and visit 2 for visits 3 and 4 . †Temporary registration for study participation. ‡Final registration for study participation. EQ-5D, Euro-Qol 5 dimension, JPAC-QOL, Japanese version of Patient Assessment of Consti- pation Quality of Life, MDS-UPDRS, Movement Disorder Society-Sponsored Revision of the Unified Parkinson's Disease Rating Scale, PDQ-39, Parkinson's Disease Questionnaire-39. 
any time during the study period. The (sub)investigator will give the eligible patient a temporary identification code and enter the patient's background in the Eligibility for Temporary Registration form of the electronic Case Report Form (eCRF; see figures 1 and 2).

The bowel movement diary and rescue medication (bisacodyl $10 \mathrm{mg}$ suppositories) will be provided to each patient at temporary registration. The (sub)investigator will instruct each patient how to complete the required items in the diary.

At visit 2, the (sub)investigator will judge the eligibility of each patient for final registration referring to the exclusion criteria (see above) and enter the background of qualified patients in the eligibility for final registration form of the eCRF. Once the patient's eligibility is confirmed, a final identification code will be assigned to the patient, who will be then allocated randomly to treatment group.

Drug allocation will be based on a stratified, permuted block method with sex as an allocation factor. Satt Co (Tokyo) will perform the allocation by preparing a drug allocation table using IWRS. The study drugs will be sealed with specific drug numbers and delivered to the individual institutional sites according to the allocation table. At the final registration of patients, the (sub)investigator will check the final identification code and the specific number of randomly allocated investigational drug for each qualified patient on the IWRS screen. The specifically numbered drug will be provided to the patient by the (sub)investigator at visit 2 and visit 3 for 2 weeks each time ( 3 tablets $\times 14$ days), as described below. The key code of the allocation table will be opened by Satt after all study data have been locked.

The patient will start the 4-week treatment period with once-daily, preprandial intake of 2 tablets of investigational drug (elobixibat $10 \mathrm{mg}$ ) or its placebo. One tablet may be added on the next day if no spontaneous bowel movement is observed during the next 24 hours. If excessive effectiveness or discomfort appears, the dosage may be decreased at the direction of the (sub)investigator or the patient's judgement. Dose augmentation from one tablet to three tablets will not be allowed. Interruption of the investigational drug due to the occurrence of an $\mathrm{AE}$ may be allowed at the direction of the (sub) investigator or the patient's judgement, but as-needed intake of the drug based on bowel movement status will not be acceptable. The drug may be restarted at the dose taken immediately before interruption or at a lower dose. Inappropriately long ( $\geq 7$ consecutive days) or frequent ( $\geq 3$ days per week during two consecutive weeks) interruption of the drug may lead to discontinuation of the patient from the study at the (sub)investigator's discretion.

\section{Rescue and concomitant medications/therapies}

Since this study is primarily planned to explore the efficacy and safety of elobixibat as a laxative in patients with PD, concomitant medications will be used throughout the study period for parkinsonism except for Duodopa pump therapy (Abbvie, North Chicago, Illinois, USA) with levodopa/carbidopa hydrate intestinal gel. Bisacodyl $10 \mathrm{mg}$ suppositories may be used once to twice daily as a rescue medication when no bowel movement is observed for 72 or more consecutive hours, unless emergency use is required.

Because of potential effects on the study results and/ or interpretation thereof, use of the following drugs or therapies as concomitant medications will be prohibited throughout the entire study period: laxatives other than elobixibat such as magnesium hydroxide, sodium picosulphate, sennosides, lubiprostone, linaclotide, polyethylene glycol (macrogol 4000), etc; oriental medicines for constipation (daiokanzoto extract, choijokito extract, daisaikoto extract, etc); medicines indicated for irritable bowel syndrome (IBS; ramosetron hydrochloride, polycarbophil calcium, trimebutine maleate, etc); supplements or over-the-counter drugs to improve the constipated condition; enema or intestinal lavage; lavage solution for colonoscopy; drugs listed in the Precaution section of the package insert of elobixibat (Goofice $5 \mathrm{mg}$ tablets) as 'with caution for concomitant use' (bile acid preparations such as ursodeoxycholic acid and chenodeoxycholic acid, aluminium-containing antacids such as sucralfate hydrate, aldioxa, etc, cholestyramine, cholestimid, digoxin, dabigatran etexylate methanesulfonate, and midazolam; other medicines or investigational drugs being used in clinical trials; non-internal therapy for constipation such as biofeedback therapy; disimpaction and Duodopa pump therapy. The patient should selfreport in his/her bowel movement diary when any of the drugs/agents/therapies listed above are used.

\section{Discontinuation of the study}

Participation of a recruited patient will be discontinued if any of the following conditions is met: judged difficult to continue due to the onset of an $\mathrm{AE}$; ineligibility confirmed after study initiation; lost to follow-up; confirmed or suspected pregnancy; voluntary withdrawal; repeated protocol violations; or any other reason for which the (sub)investigator judges that discontinuation would be necessary.

All study procedures will be discontinued if any of the following conditions is met: the Institutional Review Board of Juntendo University Hospital determines that the study should be discontinued; the appearance of safety concerns with potential impact on study progress; appearance of incidents or information that may potentially lead to impairment of ethical or scientific validity of the study; or appearance of any other incidents or information that may potentially lead to impairment of appropriateness of study conduct and/or reliability of study results.

\section{Observations and measurements}

Patient background, medical history and complications, and data of physical examinations will be collected at visit 1 (figure 2). On final judgement regarding study registration at visit 2, subjective symptoms and objective findings, 
medical history and complications, physical examinations and vital signs will be recorded for the eligible patients. Subjective symptoms and objective findings will be collected twice more at visits 3 and 4 . Physical examinations and vital signs will also be recorded at visit 4 . The JPAC-QOL,${ }^{21}$ MDS-UPDRS, ${ }^{22}$ PDQ-39 ${ }^{23}$ and EQ-5D-5L ${ }^{24} 25$ surveys and laboratory measurements (haematology and blood chemistry) will be recorded at visits 2 and 4 (figure 2).

Each patient will receive a bowel movement diary at visit 1 and will then record daily the status of bowel movements (date, stool hardness based on the Bristol scale $^{20}$ and sensation of incomplete evacuation), use of rescue medication (date, dose of bisacodyl suppositories) and concomitant medication (drugs for treatment of PD-related motor symptoms; date, drug name, dose) and treatment with non-investigational drugs and/or therapy for constipation. If the patient enters the treatment period, he/she will continue to record those items and also record daily use of the investigational drug (elobixibat or placebo; date and dose, that is, number of tablets taken) in the diary starting at visit 2 up to the end of study (visit 4). Discontinuation, if it occurs, should also be recorded in the Diary with its date and reason.

\section{Safety information}

This study will specifically focus on AEs that are suspected to have occurred in association with study conduct and include any of study conduct-associated events, impairments, deaths, infections, laboratory abnormalities and symptoms.

The (sub)investigator will examine the patient for AEs throughout the study period (figure 2) and on finding an event, record in the patient's eCRF its: name; onset date; severity (mild, moderate or severe); seriousness (non-serious or serious); defined category of seriousness (death, life-threatening, hospitalisation-initial or prolonged, disability or permanent damage, an event potentially leading to disability or permanent damage, ill condition judged as serious in reference to the aforementioned categories, or congenital anomaly or birth defect); predictability (known or unknown); action taken regarding the investigational drug (continuation, interruption or discontinuation); outcome (recovered, improving, not recovered, recovered with sequelae, died or unknown); date of outcome; causal relationship with use of the investigational drug ('not related' or grades other than 'not related'; suspected factor(s) other than the investigational drug should be recorded if judged 'not related').

\section{Study population and statistical analysis}

Based on the efficacy assessment of elobixibat vs placebo in phase 2 and phase 3 clinical trials conducted in Japanese patients with chronic constipation, ${ }^{17} 3132$ the expected effect size was calculated as 3.06 (5.66 for elobixibat $10 \mathrm{mg}$ vs 2.60 for placebo) with a common SD of 4.15 . Accordingly, we estimated a sample size of 40 patients for each group for $90 \%$ detection power at a two-sided significance level of $5 \%$. Assuming a drop-out rate of $20 \%$, we plan to recruit 100 patients with PD with chronic constipation in this study.

The full analysis set (FAS) is defined as the randomised population receiving at least one dose of the investigational drug with any measurement with regard to efficacy assessment. To ensure the robustness of study results, we also define a per-protocol set as the population after the patients are excluded from the FAS due to any of the following reasons: any of the inclusion criteria inapplicable or any of the exclusion criteria applicable; a patient receiving the investigational drug with a non-allocated drug number; a patient receiving prohibited concomitant medications or therapies; a patient deemed to be inappropriate for study participation due to low drug compliance, lost to follow-up, lack of measurements, etc. The safety analysis set is defined as a population receiving at least one dose of the investigational drug after randomisation.

Continuous variables and categorical variables will be presented as mean $\pm \mathrm{SD}$ and as frequencies and percentages, respectively. Summary statistics may include medians and quartiles, as appropriate. Comparisons will be made for primary outcomes between the elobixibat group and the placebo group by analysis of covariance using baseline values and sex as covariates. Adjusted means with 95\% CIs and $\mathrm{p}$ values will be presented at a two-sided significance level of 5\%. Within-group variations will be assessed by paired t-tests. Missing values will be imputed by the last observation carried forward (LOCF) method. Safety data will be shown as frequencies and percentages by group and individual event.

\section{Patient and public involvement}

No formal patient advisory committee was established, and there was no patient or public involvement in the design and planning of the study.

\section{Ethics and dissemination}

This study will be conducted according to the protocol that has been prepared in accordance with the Declaration of Helsinki, Clinical Trials Act of the Japan Ministry of Health, Labour and Welfare, and related laws and regulations. Each study patient will be anonymously identified by a specific identification code and hence protected against privacy invasion. The information and data of each patient will be used exclusively for the study purpose and will never be disseminated outside the study.

As a coordinating centre, SRD will share necessary information related to this study at the participating medical organisations in this study, conduct operations aimed at facilitating this study, and provide support to investigators. Data management and monitoring are conducted by Juntendo Clinical Research and Trial Center, Juntendo University Hospital. Details are specified in the Data Management Plan and Monitoring Plan.

If a serious health hazard arises due to participation in this study, coverage benefits can be received from 
insurance carried by a principal investigator, provided, however, that compensation may be reduced or not compensated if it is proven that the health hazard was caused by the research subject's own wilful act or gross negligence. In addition, if there is no causal relationship between the newly occurring health hazard and the deterioration of the originally affected disease, it is not covered by compensation. After completion of the study by each research subject, the investigators will make efforts to provide the best medical care obtained from the results of the research.

The results obtained from this study will be disseminated through an online study registry (Japan Registry of Clinical Trials). The results will also be presented at relevant scientific conferences, such as a professional congress for movement disorders and PD and in relevant medical journals.

\section{DISCUSSION AND DISSEMINATION}

PD treatment is usually focused on the amelioration of movement dysfunction. However, patients with PD have many non-motor symptoms, especially autonomic dysfunction. Chronic constipation has been considered one of the troublesome symptoms affecting the QOL of patients with PD, and it may even be life-threatening. Although fermented milk products containing probiotics and prebiotic fibres may have a favourable effect on PD-related constipation, they will not be available as laxatives. Although multiple classes of laxatives are available, there is little evidence supporting their use for PD-related constipation. An evidence-based medicine review ${ }^{1}$ recommends only three drugs/foods for PD-related constipation, including macrogol, lubiprostone and probiotics/ prebiotic fibres. Two laxatives are considered 'likely efficacious' and 'possibly useful' for the treatment of constipation in patients with PD based on the quality of randomised, controlled trials.

As mentioned above, gastrointestinal dysfunction is known to have one of the highest prevalences among the non-motor symptoms of PD. In addition, chronic constipation is known to be the earliest symptom of the prodromal phase of PD. ${ }^{33}$ Furthermore, IBS is known to be one of the prodromal gastrointestinal symptoms. ${ }^{34}$ It has been reported that chronic constipation in PD might be caused by multiple mechanisms, including decreased colonic motility, reflex inability of the pelvic floor muscles during attempted defecation and IBS. ${ }^{2}{ }^{6}$ However, the precise mechanisms underlying constipation remain unknown. Thus, the same treatment algorithm used in patients with idiopathic chronic constipation should be recommended for constipation occurring in patients with PD. ${ }^{2}$ Elobixibat is a highly potent selective IBAT inhibitor that results in excess bile acids in the colon, which is associated with increased water influx from the colon and colon motility via an interaction with TGR5. ${ }^{15}$ These action mechanisms produce favourable effects on idiopathic chronic constipation and IBS. ${ }^{17}$ Considering the effectiveness of macrogol and lubiprostone against PD-accompanying constipation, elobixibat is also expected to improve this condition. This study will provide the first evidence of whether elobixibat is a useful treatment for chronic constipation in patients with PD, as has already been demonstrated for idiopathic chronic constipation.

We believe the randomised, clinical study proposed here will be useful for expanding treatment options for PD-related constipation in an evidence-based manner.

The study findings will be presented at relevant conferences and published in a peer-reviewed journal.

\section{Author affiliations}

${ }^{1}$ Neurology, Juntendo Univerity Faculty of Medicine, Tokyo, Japan

${ }^{2}$ Neurology, Juntendo Nerima Hospital, Tokyo, Japan

${ }^{3}$ Neurology, Juntendo Urayasu Hospital, Urayasu, Japan

${ }^{4}$ Juntendo Clinical Research and Trial Center, Juntendo University Hospital, Tokyo, Japan

Correction notice This article has been corrected since it was published. The spelling error in article title (study instead of stud) has been corrected.

Acknowledgements The authors would like to thank Forte Science Communications (Tokyo, Japan) for professional assistance in preparing this manuscript.

Contributors $\mathrm{TH}, \mathrm{GO}$ and $\mathrm{NH}$ were involved in conception and trial design. TH was involved in drafting of the article. TH, GO, YS, KO, NN, JF, RN, NK, TT, YO, SS, KN, $\mathrm{HE}, \mathrm{AF}, \mathrm{AN}, \mathrm{MK}, \mathrm{DT}, \mathrm{TO}, \mathrm{HT}-\mathrm{A}, \mathrm{HK}, \mathrm{SN}$ and NH were involved in critical revision of the article for important intellectual content. All the authors were involved in final approval of the article. NY provided statistical expertise.

Funding This study will be funded by EA Pharma (Tokyo) and Mochida Pharmaceutical (Tokyo) (grant number: N/A). The two companies also provide clinical supplies, the Bowel Movement Diary and other products necessary for study conduct. It should be noted that the two companies and their employees will never be involved in any study procedures including collection, analysis or interpretation of data, ensuring (sub)investigators will be completely independent of the funders throughout study conduct.

Competing interests None declared.

Patient and public involvement Patients and/or the public were not involved in the design, or conduct, or reporting, or dissemination plans of this research.

Patient consent for publication Not applicable.

Provenance and peer review Not commissioned; externally peer reviewed.

Open access This is an open access article distributed in accordance with the Creative Commons Attribution Non Commercial (CC BY-NC 4.0) license, which permits others to distribute, remix, adapt, build upon this work non-commercially, and license their derivative works on different terms, provided the original work is properly cited, appropriate credit is given, any changes made indicated, and the use is non-commercial. See: http://creativecommons.org/licenses/by-nc/4.0/.

\section{ORCID iDs}

Taku Hatano http://orcid.org/0000-0002-6808-0444

Genko Oyama http://orcid.org/0000-0001-7985-5367

Yasushi Shimo http://orcid.org/0000-0003-1712-0845

Takashi Ogawa http://orcid.org/0000-0002-7335-0672

\section{REFERENCES}

1 Seppi K, Ray Chaudhuri K, Coelho M, et al. Update on treatments for nonmotor symptoms of Parkinson's disease-an evidence-based medicine review. Mov Disord. 2019;34:180-98.

2 Fasano A, Visanji NP, Liu LWC, et al. Gastrointestinal dysfunction in Parkinson's disease. Lancet Neurol 2015;14:625-39.

3 Pfeiffer RF. Gastrointestinal dysfunction in Parkinson's disease. The Lancet Neurology 2003;2:107-16.

4 Yu Q-J, Yu S-Y, Zuo L-J, et al. Parkinson disease with constipation: clinical features and relevant factors. Sci Rep 2018;8:567-75. 
5 Stirpe P, Hoffman M, Badiali D, et al. Constipation: an emerging risk factor for Parkinson's disease? Eur J Neurol 2016;23:1606-13.

6 Mishima T, Fukae J, Fujioka S, et al. The prevalence of constipation and irritable bowel syndrome in Parkinson's disease patients according to Rome III diagnostic criteria. J Parkinsons Dis 2017;7:353-7.

7 Seppi K, Weintraub D, Coelho M. The Movement Disorder Society Evidence-Based Medicine Review Update: Treatments for the non-motor symptoms of Parkinson's disease. Mov. Disord. 2011;26:S42-80.

8 Rossi M, Merello M, Perez-Lloret S. Management of constipation in Parkinson's disease. Expert Opin Pharmacother 2015;16:547-57.

9 The Japanese Society of Gastroenterology. Clinical practice guidelines for chronic constipation ED. by Study group of the diagnosis and treatment of chronic constipation. Tokyo Japan: Nankodo Co, Ltd, 2017.

10 Zangaglia R, Martignoni E, Glorioso M, et al. Macrogol for the treatment of constipation in Parkinson's disease. A randomized placebo-controlled study. Mov Disord. 2007;22:1239-44.

11 Ondo WG, Kenney C, Sullivan K, et al. Placebo-Controlled trial of lubiprostone for constipation associated with Parkinson disease. Neurology 2012;78:1650-4.

12 Barichella M, Pacchetti C, Bolliri C, et al. Probiotics and prebiotic fiber for constipation associated with Parkinson disease. Neurology 2016;87:1274-80.

13 Cassani E, Privitera G, Pezzoli G. Use of probiotics for the treatment of constipation in Parkinson's disease patients. Minerva Gastroenterol Dietol 2011;57:117-21.

14 Acosta A, Camilleri M. Elobixibat and its potential role in chronic idiopathic constipation. Therap Adv Gastroenterol 2014;7:167-75.

15 Appleby RN, Walters JRF. The role of bile acids in functional Gi disorders. Neurogastroenterol. Motil. 2014;26:1057-69.

16 Taniguchi S, Yano T, Imaizumi M, Elobixibat TS, et al. Elobixibat, an ileal bile acid transporter inhibitor, induces giant migrating contractions during natural defecation in conscious dogs. Neurogastroenterol Motil 2018;30:e13448.

17 Nakajima A, Seki M, Taniguchi S, et al. Safety and efficacy of elobixibat for chronic constipation: results from a randomised, double-blind, placebo-controlled, phase 3 trial and an openlabel, single-arm, phase 3 trial. Lancet Gastroenterol Hepatol 2018;3:537-47.

18 Wingate DL, Krag E, Mekhjian HS, et al. Relationships between ion and water movement in the human jejunum, ileum and colon during perfusion with bile acids. Clin Sci 1973;45:593-606.

19 Bampton PA, Dinning PG, Kennedy ML, et al. The proximal colonic motor response to rectal mechanical and chemical stimulation. Am J Physiol Gastrointest Liver Physiol 2002;282:G443-9.
20 Heaton KW, Radvan J, Cripps H, et al. Defecation frequency and timing, and stool form in the general population: a prospective study. Gut 1992;33:818-24

21 Nomura H, Agatsuma T, Mimura T. Validity and reliability of the Japanese version of the patient assessment of constipation quality of life questionnaire. J Gastroenterol 2014;49:667-73.

22 Goetz CG, Tilley BC, Shaftman SR, et al. Movement Disorder Society-sponsored revision of the Unified Parkinson's Disease Rating Scale (MDS-UPDRS): Scale presentation and clinimetric testing results. Mov. Disord. 2008;23:2129-70.

23 Peto V, Jenkinson C, Fitzpatrick R, et al. The development and validation of a short measure of functioning and well being for individuals with Parkinson's disease. Qual Life Res 1995;4:241-8.

24 Herdman M, Gudex C, Lloyd A, et al. Development and preliminary testing of the new five-level version of EQ-5D (EQ-5D-5L). Qual Life Res 2011;20:1727-36.

25 Young MK, Ng S-K, Mellick G, et al. Mapping of the PDQ-39 to EQ-5D scores in patients with Parkinson's disease. Qual Life Res 2013;22:1065-72.

26 Postuma RB, Berg D, Stern M, Stem M, et al. MDS clinical diagnostic criteria for Parkinson's disease. Mov Disord. 2015;30:1591-601.

27 Goetz CG, Poewe W, Rascol O, et al. Movement disorder Society Task force report on the Hoehn and Yahr staging scale: status and recommendations. Mov Disord 2004;19:1020-8.

28 Drossman DA, Hasler WL. Rome IV-Functional Gi disorders: disorders of gut-brain interaction. Gastroenterology 2016;150:1257-61.

29 Drossman DA. Functional gastrointestinal disorders: history, pathophysiology, clinical features, and Rome IV. Gastroenterology 2016;150:1262-79.

30 Pangman VC, Sloan J, Guse L. An examination of psychometric properties of the Mini-Mental state examination and the standardized Mini-Mental state examination: implications for clinical practice. Applied Nursing Research 2000;13:209-13.

31 Nakajima A, Seki M, Taniguchi S. Determining an optimal clinical dose of elobixibat, a novel inhibitor of the ileal bile acid transporter, in Japanese patients with chronic constipation: a phase II, multicenter, double-blind, placebo-controlled randomized clinical trial. $J$ Gastroenterol 2018;53:525-34.

32 Common Technical Document section 1.5 for Goofice $5 \mathrm{mg}$ tablets by EA Pharma Co., Ltd. (in Japanese), 2020. Available: https://www. pmda.go.jp/drugs/2018/P20180201001/index.html

33 Kalia LV, Lang AE. Parkinson's disease. The Lancet 2015;386:896-912.

34 Liu B, Sjölander A, Pedersen NL, et al. Irritable bowel syndrome and Parkinson's disease risk: register-based studies. npj Parkinsons Dis. 2021;7:5. 